\title{
Nanoparticle-labeled stem cells: a novel therapeutic vehicle
}

This article was published in the following Dove Press journal:

Clinical Pharmacology:Advances and Applications

12 March 2010

Number of times this article has been viewed

\author{
Abir O El-Sadik' \\ Afaf El-Ansary ${ }^{2}$ \\ Sherif M Sabry ${ }^{3}$ \\ 'Stem Cell Unit, Anatomy Department, \\ College of Medicine, Health Science \\ Colleges; ${ }^{2 B i o c h e m i s t r y ~ D e p a r t m e n t, ~}$ \\ Science College, King Saud University; \\ ${ }^{3}$ Anatomy Department, Faculty of \\ Medicine, Cairo University, Cairo, \\ Egypt
}

\begin{abstract}
Nanotechnology has been described as a general purpose technology. It has already generated a range of inventions and innovations. Development of nanotechnology will provide clinical medicine with a range of new diagnostic and therapeutic opportunities such as medical imaging, medical diagnosis, drug delivery, and cancer detection and management. Nanoparticles such as manganese, polystyrene, silica, titanium oxide, gold, silver, carbon, quantum dots, and iron oxide have received enormous attention in the creation of new types of analytical tools for biotechnology and life sciences. Labeling of stem cells with nanoparticles overcame the problems in homing and fixing stem cells to their desired site and guiding extension of stem cells to specific directions. Although the biologic effects of some nanoparticles have already been assessed, information on toxicity and possible mechanisms of various particle types remains inadequate. The aim of this review is to give an overview of the mechanisms of internalization and distribution of nanoparticles inside stem cells, as well as the influence of different types of nanoparticles on stem cell viability, proliferation, differentiation, and cytotoxicity, and to assess the role of nanoparticles in tracking the fate of stem cells used in tissue regeneration.
\end{abstract}

Keywords: nanoparticles, stem cells, uptake, differentiation, cytotoxicity, tracking

\section{Nanotechnology}

Nanotechnology is defined as the technology that deals with objects of nanometer $\left(10^{-9}\right.$ meter) size. It refers to the research and development which leads to the controlled manipulation and study of structures and devices in the range of 1-100 nanometers. ${ }^{1,2}$ It involves imaging, measuring, modeling, and manipulating matter at this measurement size. ${ }^{3}$ In addition, nanotechnology entails the use of atomic, molecular, and submolecular structures as core building blocks to create new products and devices. The resulting products and devices are collectively called nanomaterials. Each element is known as a nanoparticle. ${ }^{4}$

Nanoparticles can have different shapes and compositions. Their very small size imparts physical and chemical properties, particularly their high surface to volume ratio, surface tailorability, improved solubility, multifunctionality, high electrical and heat conductivity, and improved surface catalytic activity that are very different to those of the same material in the bulk form. ${ }^{5}$ These nanoparticles can be categorized into carbon-based materials, such as fullerenes and carbon nanotubes, and inorganic nanoparticles including the ones based on metal oxides (eg, zinc oxide, iron oxide, titanium dioxide, and cerium oxide), metals (gold, silver, and iron) and quantum dots (cadmium sulfide and cadmium selenide).
Correspondence:Abir O El-Sadik Stem Cell Unit, Anatomy Department, College of Medicine, Health Science Colleges, PO Box 270645 ,

Riyadh I I352, Saudi Arabia Email abgh50@yahoo.com 
Mixtures of different phases are also manufactured. In addition, these nanomaterials also present different and interesting morphologies such as spheres, tubes, rods, and prisms. Nanotechnology includes the integration of these nanoscale structures into larger material components and systems, keeping the control and construction of new and improved materials at the nanoscale. ${ }^{6}$

Nanotechnology has been described as a general purpose technology. It has already generated a range of inventions and innovations. The scientific knowledge base of nanotechnology is considered to be very interdisciplinary and multidisciplinary, combining various subfields of physics and chemistry in its extension of material sciences. ${ }^{3}$ Manufactured nanoparticles are currently used to develop products in many fields including communication, engineering, electronics, optics, energy, computer science, biology, pharmaceuticals, chemistry, cosmetics, the food industry, environmental analysis and remediation, catalysis, and material sciences. ${ }^{6-8}$

\section{Nanomedicine}

One of the major developments in nanotechnology and nanoscience studies is the production and application of nanoparticles in medical sciences, which is referred as nanomedicine. It portends a potentially endless range of applications from biomedical imaging and drug delivery to therapeutics and tissue regeneration, as well as the development of new medical products. ${ }^{9}$

Nanotechnology uses engineered materials or devices with the smallest functional organization that are able to interact with biologic systems at a molecular level. Thus, they may stimulate, respond to, and interact with target cells and tissues in order to induce desired physiologic responses while minimizing undesirable side effects. Furthermore, nanomedicine may offer ways to manipulate complex biologic systems with greater selectivity and timing than conventional pharmacologic approaches. ${ }^{10}$ The chemistry and physics of materials change more notably with size at the sub-100 nm length scales. Transcription and translation within a cellular organism occurs within the subnanometer range, and with the advent of techniques and technologies available to create materials small enough to interact selectively with biologic molecules such as nucleic acids and proteins, new opportunities to study cellular level activities are being made available. ${ }^{11}$ Nanometer-sized particles are in the same range of dimension as antibodies, membrane receptors, nucleic acids, and proteins, among other biomolecules. These biomimetic features, together with their high surface to volume ratio and the possibility of modulating their properties, make nanoparticles powerful tools for imaging, diagnosis, and therapy. ${ }^{7}$ Nanomedicine includes nanoparticles that act as biologic mimetics (eg, functionalized carbon nanotubes), "nanomachines" (eg, those made from interchangeable DNA parts and DNA scaffolds such as the octahedron and stick cube), nanofibers, and polymeric nanoconstructs as biomaterials (eg, molecular self-assembly and nanofibers of peptides and peptide-amphiphiles for tissue engineering, shape-memory polymers as molecular switches, and nanoporous membranes), and nanoscale microfabricationbased devices (eg, silicon microchips for drug release, and micromachined hollow needles and two-dimensional needle arrays from single crystal silicon), sensors, and laboratory diagnostics. $^{12}$

Development of nanotechnology will provide clinical medicine new opportunities in different areas such as medical imaging, medical diagnosis, drug delivery, and cancer detection and management.

\section{Medical imaging}

Nanoparticles can provide significant improvements in traditional biologic imaging of cells and tissues using fluorescence microscopy as well as in modern magnetic resonance imaging (MRI) of various regions of the body. ${ }^{9}$ In optical imaging techniques, nanoparticles have helped to overcome the limitations of the organic fluorophores used currently which are not photostable and have low intensity. For example, quantum dots are resistant to photobleaching and photo, chemical, and metabolic degradation. In addition, superparamagnetic iron oxide nanoparticles have already been proven to be effective in increasing contrast in magnetic imaging, serving as a complement to gadolinium-based agents, with the ability to resolve the problem of altering the relaxation times of selected types of tissue or fluid within the body such as the kidney, brain, liver, and bone marrow. Superparamagnetic iron oxide nanoparticles have been used to detect small metastases in the lymph nodes, enabling patients with localized disease to be early treated by surgery without being exposed to radiation therapy. ${ }^{13}$ Iron oxide nanocrystals have been used to monitor gene expression or detect pathologies such as cancer, brain inflammation, arthritis, or atherosclerotic plaques that would not otherwise be detectable by conventional MRI. ${ }^{14}$

\section{Medical diagnosis}

The accurate targeting and quantification of molecules indicative of cellular disorders at the single-molecule level is 
a demanding task for analysis systems. The combination of nanoparticles with other nanotechnology-based materials has the potential to address this emerging challenge and provide technologies that enable diagnosis at the level of single cells and single molecules. ${ }^{7}$ Antibodies, peptides, proteins, and nucleic acids are biologic molecules which could be linked covalently to functionalized nanoparticles which have been developed as nanoprobes for molecular detection. These functionalized nanoparticles can provide a direct rapid method of detection of infectious diseases and viruses in particular with high sensitivity. ${ }^{15,16}$

\section{Drug delivery}

Medical therapies have become more tailored to both specific diseases and to patients on an individual basis in recent years. Most pharmaceutical agents have primary targets within cells and tissues; ideally, these agents should be preferentially delivered to their sites of action within the cell. Selective subcellular delivery is likely to have considerable therapeutic benefit. ${ }^{17}$ The benefits of this type of drug delivery include efficient encapsulation of the drug, successful delivery of drug to the targeted region of the body, and successful release of the drug. Developed nanoscale particles or molecules improve the bioavailability of the drug delivery both at specific places in the body and over a longer period of time. ${ }^{18}$ Drug delivery systems are based on nanoparticles which have a mesoscopic size range of 5-200 nm, allowing their unique interaction with biologic systems at the molecular level to produce multiple advantages, eg, reduced rate of drug clearance, alteration of the pharmacokinetics and biodistribution of the drug, passage of drugs through cell membranes and into the cell cytoplasm, and regulated drug release which can avoid the tissue damage caused by some drugs. ${ }^{19}$

There are numerous nanoparticles whose unifying feature is the nanometer scale size range. These include inorganic nanoparticles (such as metals, metal oxides, and metal sulfides), polymeric nanoparticles (such as gelatins, chitosan, polylactic acid, and polyglycolic acid), dendrimers, liposomes, nanocrystals, and nanotubes. ${ }^{17}$

\section{Oncology}

Nanotechnology is playing an increasingly revolutionary role in diagnosis, prognosis, prediction, and management of targeted cancer treatment. Development of cancer therapeutic agents has the two-fold aim of achieving greater target selectivity and better delivery efficiency. To produce minimal damage to normal tissue, therapeutic drugs have been conjugated with monoclonal antibodies (mAbs) that selectively bind to antigens or receptors which are usually abundantly or uniquely expressed on the tumor cell surface. Nanoparticles have been shown to overcome both noncellular and cellularbased drug resistance and to increase selectivity of drugs toward cancer cells while reducing their toxicity toward normal tissues. ${ }^{20}$ Several types of anticancer drugs, such as liposome-based formulations of several anticancer agents (stealth liposomal doxorubicin, liposomal doxorubicin, and liposomal daunorubicin) have been approved for the treatment of metastatic breast cancer. ${ }^{21}$ Nanoparticles can deliver the therapeutic agent to the interior of the cancer cell after binding to the tumor cell surface, triggering receptormediated endocytosis. Tumor-targeting ligands, such as antibodies, growth factors, or cytokines, have been used to facilitate the uptake of carriers into target cells. ${ }^{22}$

\section{Nanomedicine and stem cells}

Stem cell therapy is a rapidly evolving area of research in regenerative medicine. ${ }^{23,24}$ Mesenchymal stem cells have received considerable attention in the field of cell therapy because of their ability to differentiate into various tissues of mesenchymal origin (eg, bone, cartilage, fat, muscle, marrow stroma, tendon, ligament, and other connective tissues), ${ }^{25}$ making them excellent candidates for cell and tissue replacement therapies. ${ }^{26,27}$ However, there are still some obstacles in targeting stem cell therapy, including the problems in homing and fixing stem cells to their desired site and guiding extension of stem cells in specific directions. Nanoparticles have been used to overcome these challenges in the therapeutic application of stem cells. ${ }^{28}$

Despite the wide range of applications of nanoparticles in the stem cell field, there is a dire lack of information concerning the impact of manufactured nanomaterials on human health and the environment. ${ }^{29}$ This review reviews the most recent influences of nanoparticles on stem cell behavior. Biocompatibility of nanoparticles is the prerequisite for their applications in biomedicine, but this can be misleading due to the absence of criteria for evaluating their potential toxicity. ${ }^{30}$

More than 10 million nanoparticles are ingested per person every day. ${ }^{31}$ The nanoparticles are small enough to penetrate even very small capillaries throughout the body, and can pass through biologic membranes, affecting cell function anywhere in the body. This consideration is of importance for stem cells, where the effects of nanoparticles on the potential for self-renewal and differentiation of stem cells is unknown. Data available from toxicity studies of nanoparticles, particularly in stem cells, are limited and 
the molecular mechanisms of nanoparticle toxicity are still poorly understood. ${ }^{29}$

Clinical and experimental studies indicate that a small size, a large surface area, and the ability to generate reactive oxygen species contributes to the potential of nanoparticles to induce cell injury. ${ }^{32}$ Braydich-Stolle et $\mathrm{al}^{29}$ demonstrated that the cytotoxicity of nanoparticles is concentration-dependent. Dramatic changes occurred when cadmium oxide was added to germ-line stem cells. Within 48 hours of exposure at cadmium oxide concentrations of $1 \mu \mathrm{g} / \mathrm{mL}$, stem cells were shown to shrink and become irregular, with significant inhibition of mitochondrial function. At concentrations of cadmium oxide higher than $5 \mu \mathrm{g} / \mathrm{mL}$, stem cells became necrotic and detached from the culture dishes.

Development of new techniques to show accurate correlations between in vitro and in vivo studies is imperative to portray accurately nanoparticle effects. Moreover, toxicity studies are critical to establish the full in vivo potential of nanomedicine. Understanding the physicochemical, molecular, and physiologic processes of nanoparticles is important for nanomedicine to become a reliable and sustainable treatment modality. ${ }^{33}$

\section{Cellular uptake of nanoparticles by stem cells}

Stem cells utilize various endocytic mechanisms for the uptake of nanoparticles, including phagocytosis, macropinocytosis, clathrin-dependent endocytosis, caveolae-dependent uptake, and non-clathrin/non-caveolae endocytosis. ${ }^{34}$

With regard to silica nanoparticles, mesoporous silica conjugated with fluorescein isothiocyanate was efficiently internalized into human bone marrow mesenchymal stem cells even in short-term incubation. ${ }^{26}$ The uptake of mesoporous silica nanoparticles was time- and concentrationdependent. Uptake began as early as 10 minutes after incubation and was relatively rapid within the first 1-2 hours of incubation, and a saturation uptake was achieved after 4 hours of incubation. Chung et $\mathrm{al}^{35}$ demonstrated that internalization of mesoporous silica nanoparticles was mediated by both clathrin- and actin-dependent endocytosis. Huang et $\mathrm{al}^{30}$ found that dynamic polymerization of actin filaments has a central role in the uptake of mesoporous silica nanoparticles, and found that inhibition of actin polymerization by cytochalasin D decreased their uptake. Ito et $\mathrm{al}^{36}$ found that uptake of magnetic iron oxide cationic liposome nanoparticles began rapidly, and maximum uptake was achieved after 4 hours. The uptake of superparamagnetic iron oxide was shown to be mediated via endocytosis in human mesenchymal stem cells. ${ }^{25}$ Numerous studies have established a variety of techniques to deliver quantum dots into cells, such as microinjection, endocytic compartment, electroporation, liposome-mediated transfection, and special peptide delivery. ${ }^{37}$ Chang et al ${ }^{38}$ investigated cytoskeletal reorganization after the uptake of quantum dots which revealed the formation of wide and flat leading lamellipodia filled with a dense actin network in cells one day after addition. Liu et $\mathrm{a}^{39}$ studied the endocytic mechanisms of $100 \mathrm{~nm}$ nanodiamond particles in stem cells and found that these particles were taken into the cells by macropinocytosis and clathrin-mediated endocytosis. The macropinocytosis was mediated by filopodia from cell membrane extension and cell-surface ruffling and invagination. It is possible that the mechanism of nanoparticle uptake by stem cells is influenced by the type, size, shape, concentration, and surface modification of the nanoparticles.

\section{Location and distribution of nanoparticles in stem cells}

After internalization of nanoparticles into stem cells, they become located in vesicles inside the cytoplasm. For instance, polystyrene particles ranging from $40 \mathrm{~nm}$ to over $120 \mathrm{~nm}$ were found scattered in the cell cytoplasm and cytoplasmic extension pseudopods. ${ }^{31}$ The nanoparticles were also located in the perinuclear region, forming rings around the cell nucleus. Nanodiamond particles of $100 \mathrm{~nm}$ were accumulated to develop a single nanodiamond cluster in a cytoplasmic vesicle, which contained several nanodiamond particles but they did not enter the nucleus. ${ }^{39}$ In addition, after uptake into stem cells, superparamagnetic iron oxide nanoparticles were located inside the cytoplasm and vesicles, and could then be transferred to lysosomes, in which degradation of the nanoparticles could occur and free iron be released into the cytoplasm. ${ }^{40,41}$ In the quantum dots studies, Chang et $\mathrm{al}^{37}$ found that delivered quantum dots had escaped from lysosome degradation at the beginning of the uptake. At 21 days, lysosome expression was enhanced and almost all the cellular quantum dots were observed in lysosome vesicles at this time.

\section{Effects on viability, morphology, and proliferation of stem cells}

The influences of different types of nanoparticles on the behavior of stem cells have been evaluated by several studies. Huang et $\mathrm{al}^{26}$ and Chung et $\mathrm{al}^{35}$ concluded that internalization of mesoporous silica nanoparticles conjugated with fluorescein isothiocyanate in human bone marrow 
mesenchymal stem cells did not affect stem cell viability and proliferation. They proved that silica nanoparticles could escape from endolysosomal vesicles and retained their architectonic integrity after internalization. In addition, Huang et $\mathrm{al}^{30}$ reported that silica nanoparticles can enhance actin polymerization induction in mesenchymal stem cells.

Ito et $\mathrm{al}^{36}$ demonstrated that magnetic nanoparticles could be used to expand mesenchymal stem cells efficiently for clinical application. Mesenchymal stem cells incubated in osteogenic medium with magnetite iron oxide cationic liposomes changed their shape from fibroblastic to polygonal and formed calcium nodules. When mesenchymal stem cells were seeded at high density using magnetite cationic liposomes, there was a five-fold increase in the number of cells, compared with numbers seen in culture prepared without magnetite cationic liposomes. The survival rate of stem cells cultured in a medium containing superparamagnetic iron oxide was very high (97\%-99\%) indicating that superparamagnetic iron oxide did not affect stem cell viability. ${ }^{23,25,41,42}$ These observations suggest a promising role for nanomedicine in stem cell-related therapies. Selection of type and concentration of nanoparticles is critically important.

Kea et $\mathrm{al}^{43}$ showed for the first time, that neural stem cells derived from bone marrow stroma of rhesus monkeys could be labeled in vitro with the superparamagnetic iron oxide contrast agent Feridex and Poly-L-Lysine without affecting morphology, cell cycle, telomerase activity, proliferation, and differentiation ability of the labeled cells. Huang et $\mathrm{al}^{24}$ detected that ferucarbotran nanoparticles could promote the growth of human mesenchymal stem cells due to their ability to diminish intracellular $\mathrm{H}_{2} \mathrm{O}_{2}$ through intrinsic peroxidaselike activity. They reported that ferucarbotran could accelerate cell cycle progression mediated by free iron released from lysosomal degradation and involved alteration of iron on the expression of protein regulators of the cell cycle. Jung et $\mathrm{al}^{44}$ studied the topologic and physical properties of the fibrinpoly(lactide-caprolactone) scaffolds. These nanoparticles were designed to improve stem cell seeding efficiency and cell in-growth, while enabling maintenance of the mechanical integrity and efficient delivery of mechanical signals to adherent cells and lead to a stronger biologic response.

On the other hand, embryonic stem cells cultured in low concentration $(0.4 \mathrm{million} / \mathrm{mL})$ polystyrene nanoparticles became mostly columnar and embryoid bodyshaped. However, in high concentration (12.2 million $/ \mathrm{mL}$ ) nanoparticles, they became fibroblast-like and less squamous types. At the 24-hour time point, the presence of the nanoparticles reduced viability of the embryonic stem cells by $40 \%$ and $30 \%$ in the low versus high relative concentration of nanoparticles, respectively. After six days of culture, there was a significantly smaller cell surface area associated with embryonic stem cells exposed to polystyrene nanoparticles compared with controls which was not concentration-dependent. This effect indicated the disruption of cytoskeletal development. In contrast, the nanoparticles did not have an effect on the size of the embryonic stem cell nucleus. ${ }^{31}$

\section{Effect on stem cell differentiation}

It is still controversial whether magnetic labeling with superparamagnetic iron oxide inhibits chondrogenic differentiation of mesenchymal stem cells. ${ }^{45}$ Meanwhile, Heymer et al, ${ }^{25}$ Delcroix et $\mathrm{al}^{23}$ and Wang et $\mathrm{al}^{42}$ detected that superparamagnetic iron oxide did not affect human mesenchymal stem cells undergoing adipogenic, osteogenic, or myogenic differentiation. In addition, naringin nanoparticles in concentrations $(1-100 \mu \mathrm{g} / \mathrm{mL})$ could enhance the proliferation and osteogenic differentiation of human bone mesenchymal stem cells. ${ }^{46} \mathrm{Au}$ et $\mathrm{al}^{47}$ detected that superparamagnetic iron oxide labeling did not affect the calcium-handling properties of cardiomyocytes derived from embryonic stem cells. Moreover, transplantation of superparamagnetic iron oxide labeled embryonic stem cells via direct injection into infarcted myocardium resulted in significant improvement in heart function. These findings demonstrated the feasibility of in vivo tracking of embryonic stem cells using superparamagnetic iron oxide labeling and cardiac MRI, without affecting the cardiac differentiation potential and functional properties of embryonic stem cells.

In their study of silica nanoparticles, Huang et $\mathrm{al}^{30}$ concluded that the uptake of mesoporous silica nanoparticles into human mesenchymal stem cells did not affect regular osteogenic differentiation of these cells. In vitro osteogenesis was successfully induced in the mesenchymal stem cells in highly chondrogenic synovium after the uptake of mesoporous silica nanoparticles. ${ }^{48}$

Fibrin-polylactide-caprolactone (PLCL) nanoparticles facilitated the upregulation of chondrogenesis marker genes and chondrogenic differentiation in vitro. The nanoparticle-fibrin-PLCL complex had chemical and physiologic characteristics designed to induce chondrogenic differentiation to sustain their lineage in the complex. In vitro and in vivo studies revealed that the complex developed effectively sustained chondrogenic differentiation and led to significant enhancement of the chondral extracellular matrix deposition on human adipogenic stem cells-fibrin-PLCL nanoparticle 
complex constructs. This complex will be useful for in situ cartilage tissue engineering using human stem cells. ${ }^{44}$

In contrast, Oliveira et $\mathrm{al}^{49}$ showed that direct exposure to biphasic calcium phosphate particles impaired human mesenchymal stem cell osteogenic differentiation and bone matrix mineralization. They added that exposure to calcium-deficient apatite particles severely impaired human mesenchymal stem cell osteogenic maturation owing to the uptake of $\mathrm{Ca}^{2+}$ from the culture media.

Hussain et $\mathrm{al}^{50}$ demonstrated that the level of cytotoxicity of nanoparticle-labeled stem cells depended on the type of the nanoparticles. Silver nanoparticles were detected to be the most toxic, and this cytotoxicity was mediated through oxidative stress. Carbon nanomaterials significantly impaired phagocytosis and induced cell death in alveolar macrophages. ${ }^{51}$ Polystrene nanoparticles influenced viability and differentiation of embryonic stem cells to a minor extent. $^{31}$

\section{In vivo tracking of nanoparticle-labeled stem cells}

Delivery of sufficient stem cells to the regions of interest with the smallest number of cells to untargeted regions is a prerequisite for maximizing the therapeutic benefits and minimizing possible unwanted effects of stem cell therapy. To monitor distribution, density, proliferation, and transdifferentiation of engrafted stem cells, reliable cellular imaging techniques are required. ${ }^{42}$

Tracking the fate of stem cells employed in developmental biology and tissue regeneration is essential to understand how these cells participate in tissue repair. Inorganic probes with special physical properties have been developed using different systems of detectors such as quantum dots, light-emitting semiconductors, mesoporous silica nanoparticles, and superparamagnetic nanoparticle iron oxide. ${ }^{38}$

Huang et $\mathrm{al}^{26}$ concluded that the advantages of biocompatibility, durability, and higher efficiency in internalization of mesoporous silica nanoparticles made them a better vector for stem cell tracking than others currently used. Silica coating and polyethylene glycolation confers additional stability and biocompatibility to the magnetic ferrite nanoparticles. Organic dyes incorporated into the silica shell enabled the detection of multifunctional nanoparticle fluorescence.

Sykova et $\mathrm{al}^{52}$ tracked the fate of embryonic stem cells and bone marrow mesenchymal stem cells labeled with iron oxide nanoparticles in rats with cortical or spinal cord lesions. Cells were either grafted intracerebrally and contralaterally to a cortical photochemical lesion, or injected intravenously. During the first post-transplantation week, grafted cells migrated to the lesion site in the cortex or spinal cord and were visible in the lesion on MR images as a hypointense signal, persisting for more than 30 days. Their studies demonstrated that MRI of grafted stem cells labeled with iron oxide nanoparticles is a useful method for evaluating cellular migration toward a lesion site. ${ }^{23,52}$ Furthermore, Kea et $\mathrm{al}^{43}$ autografted Feridex-labeled neural stem cells derived from bone marrow stroma cells into the striatum of the rhesus monkey brain. They found that these cells survived, differentiated, and were incorporated into the brain, and could be reliably tracked using MRI. Concerning articular cartilage repair, labelling with superparamagnetic nanoparticle iron oxide had great potential to visualise human mesenchymal stem cells and track their migration after transplantation for articular cartilage repair with MRI following intra-articular injection. ${ }^{25,47}$ Using MRI, Lee et al ${ }^{53}$ successfully tracked the multifunctional nanoparticle-labeled mesenchymal stem cells in the hearts of mice at seven days after induction of myocardial infarction. Thus, being able to monitor the fate of transplanted stem cells in vivo is crucial for developing successful cell therapies. MRI has afforded superb spatial resolution and repeated noninvasive imaging of magnetically nanoparticle-labeled stem cells, and appears to be the best modality for cell imaging. ${ }^{24,52}$

\section{Conclusion}

Internalization of nanoparticles into stem cells had a significant influence on diverse aspects of cell activities. Viability, proliferation, differentiation, cytotoxicity, and in vivo tracking of stem cells labeled with nanoparticles have been shown to be type- and concentration-dependent. Therefore, more studies are needed to investigate the effects of different nanoparticles in directing stem cell behavior and should provide valuable information about the impact of nanoparticles in diagnostic and therapeutic applications.

\section{Disclosures}

The authors report no conflicts of interest in this work.

\section{References}

1. Abraham AM, Kannangai R, Sridharan G. Nanotechnology: A new frontier in virus detection in clinical practice. Indian J Microbiol. 2008;26:297-301.

2. Gao J, Xu B. Applications of nanomaterials inside cells. Nanotoday. 2009;4:37-51. 
3. Nikulainen T, Palmberg C. Transferring science-based technologies to industry. Does nanotechnology make a difference? Technovation. 2010;30:3-11.

4. Staggers N, McCasky T, Brazelton N, et al. Nanotechnology: The coming revolution and its implications for consumers, clinicians, and informatics. Nurs Outlook. 2008;56:268-274.

5. Azzazy H, Mansour M. In vitro diagnostic prospects of nanoparticles. Clin Chim Acta. 2009;403:1-8.

6. Ju-Nam Y, Lead JR. Manufacured nanoparticles: An overview of their chemistry, interactions and potential environmental implications. Sci Total Environ. 2008;400:396-414.

7. Sanvicens N, Marco MP. Multifunctional nanoparticles - properties and prospects for their use in human medicine. Trends Biotechnol. 2008;26:425-433.

8. Murday JS, Siegel RW, Stein J, Wright JF. Translational nanomedicine: Status assessment and opportunities. Nanomedicine. 2009;5: 251-273.

9. Murthy SK. Nanoparticles in modern medicine: State of the art and future challenges. Int J Nanomedicine. 2007;2:129-141.

10. Modi G, Pillay V, Choonara YE, et al. Nanotechnological applications for the treatment of neurodegenerative disorders. Prog Neurobiol. 2009;88:272-285.

11. Suh WH, Suh Y, Stucky GD. Multifunctional nanosystems at the interface of physical and life sciences. Nanotoday. 2009;4:27-36

12. Moghimi SM, Hunter AC, Murray JC. Nanomedicine: Current status and future prospects. FASEB J. 2005;19:311-330.

13. Harisinghani MG, Barentsz J, Hahn PF, et al. Noninvasive detection of clinically occult lymph node metastases in prostate cancer. $N$ Engl J Med. 2003;348:2491-2499.

14. Saleh A, Schroeter M, Jinkmanns C, et al. In vivo MRI of brain inflammation in human ischaemic stroke. Brain. 2004;127: 1670-1677.

15. Tang S, Zhao J, Storhoff JJ, et al. Nanoparticle-based biobarcode amplification assay (BCA) for sensitive and early detection of human immunodeficiency type 1 capsid (p24) antigen. J Acquir Immune Defic Syndr. 2007;46:231-237.

16. Tripp RA, Alvarez R, Anderson B, et al. Bioconjugated nanoparticle detection of respiratory syncytial virus infection. Int J Nanomedicine. 2007;2:117-124.

17. Faraji AH, Wipf P. Nanoparticles in cellular drug delivery. Bioorg Med Chem. 2009;17:2950-2962.

18. Cavalcanti A, Shirinzadeh B, Freitas RA, et al. Nanorobot architecture for medical target identification. Nanotechnology. 2008;19: 15103-15118.

19. Allen TM, Cullis PR. Drug delivery systems: Entering the mainstream. Science. 2004;303:1818-1822.

20. Wang X, Yang L, Chen Z. Application of nanotechnology in cancer therapy and imaging. CA Cancer J Clin. 2008;58:97-110.

21. Allison SD. Liposomal drug delivery. J Infus Nurs. 2007;30:89-95.

22. Guillemard V, Saragovi HU. Novel approaches for targeted cancer therapy. Curr Cancer Drug Targets. 2004;4:313-326.

23. Delcroix GJ, Jacquart M, Lemaire L, et al. Mesenchymal and neural stem cells labeled with HEDP-coated SPIO nanoparticles: In vitro characterization and migration potential in rat brain. Brain Res. 2009;1255:18-31.

24. Huang D, Hsiao J, Chen Y, et al. The promotion of human mesenchymal stem cell proliferation by superparamagnetic iron oxide nanoparticles. Biomaterials. 2009;30:3645-3651.

25. Heymer A, Haddad D, Weber M, et al. Iron oxide labelling of human mesenchymal stem cells in collagen hydrogels for articular cartilage repair. Biomaterials. 2008;29:1473-1483.

26. Huang D, Hung Y, Ko B, et al. Highly efficient cellular labeling of mesoporous nanoparticles in human mesenchymal stem cells: Implication for stem cell tracking. FASEB J. 2005;19:2014-2016.

27. Ami D, Neri T, Natalello A, et al. Embryonic stem cell differentiation studied by FT-IR spectroscopy. Biochim Biophys Acta. 2008;1783: 98-106.
28. Park TH, Lee HJ, Kim JA, et al. Application of magnetic bio-nanoparticles to the control of stem cell behavior. Biotechnology. 2007;131:S65.

29. Braydich-Stolle L, Hussain S, Schlager JJ, et al. In vitro cytotoxicity of nanoparticles in mammalian germline stem cells. Toxicol Sci. 2005;88:412-419.

30. Huang D, Chung T, Hung Y, et al. Internalization of mesoporous silica nanoparticles induces transient but not sufficient osteogenic signals in human mesenchymal stem cells. Toxicol Appl Pharmacol. 2008;231:208-215.

31. Tran DMD, Lin C, Ota BS, et al. Influence of nanoparticles on morphological differentiation of mouse embryonic stem cells. Fertil Steril. 2007;87:965-970.

32. Nel A, Xia T, Mädler L, Li N. Toxic potential of materials at the nanolevel. Science. 2006;311:622-627.

33. El-Ansary A, Al-Daihan S. Review article on the toxicity of therapeutically used nanoparticles: An overview. J Toxicol. Epub 2009 Jan 25.

34. Conner SD, Schmid SL. Regulated portals of entry into the cell. Nature. 2003;422:37-44

35. Chung T, Wu S, Yao M, et al. The effect of surface charge on the uptake and biological function of mesoporous silica nanoparticles in 3T3-L1 cells and human mesenchymal stem cells. Biomaterials. 2007;28:2959-2966

36. Ito A, Hibino $\mathrm{E}$, Honda $\mathrm{H}$, et al. A new methodology of mesenchymal stem cell expansion using magnetic nanoparticle. Biochemical and Engineering J. 2004;20:119-125.

37. Chang J, Su H, Hsu S. The use of peptide-delivery to protect human adipose-derived adult stem cells from damage caused by the internalization of quantum dots. Biomaterials. 2008;29:925-936.

38. Chang J, Hsu S, Su H. The regulation of the gap junction of human mesenchymal stem cells through the internalization of quantum dots. Biomaterials. 2009;30:1937-1946.

39. Liu K, Wang C, Cheng C, et al. Endocytic carboxylated nanodiamond for the labeling and tracking of cell division and differentiation in cancer and stem cells. Biomaterials. 2009;30:4249-4259.

40. Arbab AS, Wilson LB, Ashari P, et al. A model of lysosomal metabolism of dextran coated superparamagnetic iron oxide (SPIO) nanoparticles: Implications for cellular magnetic resonance imaging. NMR Biomed. 2005; 18:383-389.

41. Jing X, Yang L, Duan X, et al. In vivo MR imaging tracking of magnetic iron oxide nanoparticle labeled, engineered, autologous bone marrow mesenchymal stem cells following intra-articular injection. Joint Bone Spine. 2008;75:432-438.

42. Wang L, Deng J, Wang J, et al. Superparamagnetic iron oxide does not affect the viability and function of adipose-derived stem cells, and superparamagnetic iron oxide-enhanced magnetic resonance imaging identifies viable cells. Magn Reson Imaging. 2009;27:108-119.

43. Kea $\mathrm{Y}, \mathrm{Hu} \mathrm{C}$, Jianga $\mathrm{X}$, et al. In vivo magnetic resonance tracking of Feridex-labeled bone marrow-derived neural stem cells after autologous transplantation in rhesus monkey. J Neurosci Methods. 2009;179: 45-50.

44. Jung Y, Chung Y, Kim SH, et al. In situ chondrogenic differentiation of human adipose tissue-derived stem cells in a TGF-b1 loaded fibrinpoly (lactide-caprolactone) nanoparticulate. Biomaterials. 2009;30: 4657-4664.

45. Kostura L, Kraitchman DL, Mackay AM, et al. Feridex labeling of mesenchymal stem cells inhibits chondrogenesis but not adipogenesis or osteogenesis. NMR Biomed. 2004;17:513-517.

46. Zhang P, Dai K, Yan S. Effects of naringin on the proliferation and osteogenic differentiation of human bone mesenchymal stem cell. Eur J Pharmacol. 2009;607:1-5.

47. Au K, Liao S, Lee Y, et al. Effects of iron oxide nanoparticles on cardiac differentiation of embryonic stem cells. Biochem Biophys Res Commun. 2009;379:898-903.

48. Shi X, Wang Y, Varshney RR, et al. In-vitro osteogenesis of synovium stem cells induced by controlled release of bisphosphate additives from microspherical mesoporous silica composite. Biomaterials. 2009;30:3996-4005. 
49. Oliveira JM, Sousa RA, Kotobuki N, et al. The osteogenic differentiation of rat bone marrow stromal cells cultured with dexamethasone-loaded carboxymethylchitosan/poly(amidoamine) dendrimer nanoparticles. Biomaterials. 2009;30:804-813.

50. Hussain SM, Hess KL, Gearhart JM, et al. In vitro toxicity of nanoparticles in BRL 3A rat liver cells. Toxicol In Vitro. 2005;19: 975-983.

51. Jia G, Wang H, Yan L, et al. Cytotoxicity of carbon nanomaterials: single-wall nanotube, multi-wall nanotube, and fullerene. Environ Sci Technol. 2005;39:1378-1383.
52. Sykova $\mathrm{E}$, Jendelova P. In vivo tracking of stem cells in brain and spinal cord injury. Prog Brain Research. 2007;161:367-383.

53. Lee K, Park C, Moon H, et al. Magnetic resonance tracking of multifunctional nanoparticle-labeled mouse mesenchymal stem cells in a mouse model of myocardial infarction. Current Applied Physics. 2009;9(Suppl 1):S12-S14.

\section{Publish your work in this journal}

Clinical Pharmacology: Advances and Applications is an international, peer-reviewed, open access journal publishing original research, reports, reviews and commentaries on all areas of drug experience in humans The manuscript management system is completely online and includes a very quick and fair peer-review system, which is all easy to use.

Submit your manuscript here: http://www.dovepress.com/clinical-pharmacology-advances-and-applications-journal
Visit http://www.dovepress.com/testimonials.php to read real quotes from published authors. 